\section{The Initial Steps in Neonatal Resuscitation}

We read with interest the randomized control trial by Kumar, et al. [1] and would like to make some observations. Neonatal Resuscitation program as per the International Liaison Committee on Resuscitation (ILCOR) guidelines had made it clear that the initial steps should include, in order, positioning the baby under radiant warmer, then suctioning if there is a lot of secretions, and after that only drying and gentle stimulation, if needed. The process of rubbing the baby's back while drying, itself is a good stimulant for $90 \%$ of the babies to make a good cry and establish respiration. Certain points require consideration:

1. The reason this RCT did not pick up the difference is that the study was looking at composite outcome of admission temperature in the NICU and respiratory distress at 6 hours of age.

2. In the study only $5.2 \%$ in the first group (suctioning before drying) died, while $11.7 \%$ died in the second group (drying before suctioning) [RR $(95 \% \mathrm{CI}) 0.44(0.14-0.38)]$. This is an eye opener that depressed sick babies require suctioning first and then only drying.

3. The babies were transported to NICU without any additional source of heat which is not acceptable. Even years back, we have the consensus that the baby should be transported in a transport incubator, or a warmer or phase exchange material like Embrace or at least swaddling the baby well. Ideally babies temperature should have been recorded in the delivery room itself.

To bring uniformity and consistency among health professionals and to avoid confusion in implementation of NRP guidelines, let us follow a scientific and common sense approach which is in agreement with the standard guidelines. So in the initial steps of resuscitation of a depressed neonate (apneic/gasping/ decreased tone) let us follow the standard guidelines of NRP namely, positioning under the warmer, suctioning if needed, drying and removing the wet linen, followed by gentle stimulation or evaluation for respiration and heart rate [2].

\section{Emeritus Professor, Department of Neonatology, SAT Hospital, Medical College, Trivandrum, Kerala. \\ drpmc50@gmail.com}

\section{REFERENCES}

1. Kumar A, Yadav RP, Basu S, Singh TB. Suctioning first or drying first during delivery room resuscitation: A randomized controlled trial. Indian Pediatr. 2021:58:25-9.
2. Wyckoff MH, Aziz K, Escobedo MB, et al. Part 13: Neonatal Resuscitation: 2015 American Heart Association Guidelines Update for Cardiopulmonary Resuscitation and Emergency Cardiovascular Care. Circulation.2015;132: S543-60.

\section{AUTHORS' REPLY}

1. The sequence of performing suctioning and drying during initial steps of delivery room resuscitation is not evidencebased. The purpose of suctioning (clearing airway) is to prevent aspiration of secretions and respiratory distress while drying helps to prevent hypothermia. In our study, we compared the sequence of the two approaches (suctioning first versus drying first). We feel that without using the composite outcome of admission hypothermia or respiratory distress, we would have favored one group over the other, compromising the validity of the trial. Separate analysis of the incidences of admission hypothermia or respiratory distress did not show any significant difference between the two groups.

2. Relative risk should be interpreted in relation to the $95 \%$ confidence interval, which was quite wide (0.14-1.38). Based on our findings, there was no significant difference between the two approaches. We therefore differ with the reader that depressed newborns should always be suctioned first followed by drying. Interestingly, the upcoming eighth edition of NRP by the American Academy of Pediatrics has reordered the sequence of initial steps (drying first and suctioning later, if needed) to better reflect the common practice [2]. It appears that the proposed change is based on expert opinion rather than any evidence. In fact, our study provides evidence to this change of practice.

3. We agree to the concern of the authors. Our NICU is close to both labor room and maternity operation theatre. Babies are transported to NICU well swaddled. We do not have transport incubator. Currently we are using Embrace to maintain temperature during transportation. However, its use does not entirely eliminate the problem of admission hypothermia.

\section{REFERENCES}

Ashok Kumar

Department of Pediatrics, IMS-BHU,

Varanasi, UP.

ashokkumarbhu@hotmail.com

1. Kumar A, Yadav RP, Basu S, Singh TB. Suctioning first or drying first during delivery room resuscitation: A randomized controlled trial. Indian Pediatr. 2021;58:25-9.

2. NRP 8th Edition Busy People Update \#1 - December 2020. Accessed March 01, 2021. Available from: https://downloads.aap. org/AAP/PDF/NRP\%208th\%20Edition\%20Busy\%20People\% 20Update\%20.pdf 